\title{
Flexible Temperature Microsensor for Application of High-Intensity Focused Ultrasound
}

\author{
Bin Xu, Gang Tang, ${ }^{*}$ Cui-qun He, and Xiao-xiao Yan \\ Jiangxi Province Key Laboratory of Precision Drive and Control, Department of Mechanical and Electrical Engineering, \\ Nanchang Institute of Technology, No. 289, Tianxiang Road, Nanchang, Jiangxi 330099, China
}

(Received July 31, 2017; accepted October 24, 2017)

Keywords: microsensor, flexible sensor, temperature measurement, high-intensity focused ultrasound

A flexible temperature microsensor based on a polyimide thin-film substrate is presented. The polyimide thin-film substrate can achieve good contact with skin during tumor ablation, and it has a high ultrasonic propagation rate when ultrasound is passed though the thin substrate. A serpentine structure of platinum film is designed as the sensing element, and the sensor is fabricated using microfabrication technology. The characteristics of the sensor were studied in the temperature range from 25 to $100{ }^{\circ} \mathrm{C}$. The testing results show that there is a good linear relationship between resistance and temperature, and the temperature coefficient of resistance $(T C R)$ of the flexible temperature microsensor is $2035 \mathrm{ppm} /{ }^{\circ} \mathrm{C}$. The current-voltage $(I-V)$ curve shows that the temperature can be precisely detected when the drive current is lower than $20 \mathrm{~mA}$. Impedance testing results show that impedance does not change with the frequency of the $\mathrm{AC}$ current. In addition, the impedance of the sensor under AC conditions has the same value as the resistance under DC conditions, indicating that the performance measured under DC current is the same as that measured under AC current. Such a flexible microsensor can be used to measure the temperature distribution for high-intensity focused ultrasound (HIFU).

\section{Introduction}

High-intensity focused ultrasound (HIFU) has been extensively studied during the past decade as a clinical alternative for thermal ablation of tumor tissue. ${ }^{(1,2)}$ Volumetric sonication efficiently enlarges the ablated volume per sonication but remains limited by the increasing temperature induced in the skin and fat layers. To avoid thermal lesion in the skin layer, it is necessary to obtain the temperature distribution on the skin. ${ }^{(3)}$ A temperature sensor is proposed to measure this temperature distribution. A schematic diagram of the method is shown in Fig. 1. The transducer generates ultrasonic beams focused into the target to ablate the tumor.

To be in good contact with skin and decrease ultrasonic intensity loss through the sensor during temperature measurement, a thin substrate is suitable as the substrate for the sensor. Traditional materials such as silicon and glass are fragile and have other poor mechanical properties when they are too thin. A sensor fabricated on a parylene substrate has been reported ${ }^{(4)}$ as having a linear relationship between resistance and temperature. However, the parylene substrate is not resistant to high temperatures, especially the bonding temperature for the lead wire, which

"Corresponding author: e-mail: tanggang@nit.edu.cn

http://dx.doi.org/10.18494/SAM.2017.1714 


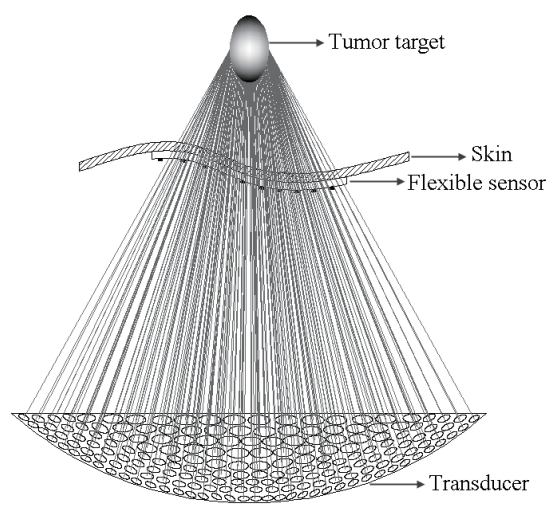

Fig. 1. Schematic diagram of temperature measurement on skin.

is higher than $150{ }^{\circ} \mathrm{C}$. Trung et al. fabricated an all-elastomeric temperature sensor array on polydimethylsiloxane (PDMS), that can be attached as a patch to human skin. ${ }^{(5)}$ PDMS has a small modulus of elasticity, and the displacement is large under small force. The resistance of metal film deposited on a PDMS substrate varies largely, because, under tension conditions, the particles of metal film move away from each other, while in compression, they become closer to each other. ${ }^{(6)}$ Polyimide overcomes the shortcoming of parylene and provides many advantages, such as good biocompatibility, good electrical insulation, high-temperature resistance $\left(400{ }^{\circ} \mathrm{C}\right)$, high mechanical flexibility, ${ }^{(7-11)}$ and high ultrasonic propagation rate. ${ }^{(12)}$ Platinum is selected as the sensor sensitive material, as it has a good thermal response that corresponds linearly to temperature. ${ }^{(13-15)}$ There is much interest in the development of polyimide-based devices for medical applications, which include implantable microelectrodes ${ }^{(16)}$ for neural prostheses, ${ }^{(17)}$ micro-hotplates and microheater arrays, ${ }^{(18,19)}$ and bolometers. ${ }^{(20)}$ To our knowledge, no one has addressed the fabrication of a temperature microsensor to measure the temperature distribution on the skin during ultrasound exposure. Microfabrication technology is introduced herein for a temperature microsensor that can measure the temperature distribution precisely and quickly. The thermal ablation temperature for tumor cells is above $43{ }^{\circ} \mathrm{C}$, and the safe temperature for skin is below $50{ }^{\circ} \mathrm{C}$. To efficiently ablate tumor cells, the selected temperature for HIFU must be higher than $43{ }^{\circ} \mathrm{C}$. Thus, a sensor for the temperature range between 25 and $100{ }^{\circ} \mathrm{C}$ is proposed.

In this study, a thin flexible temperature microsensor based on a polyimide substrate for HIFU applications is proposed and fabricated. The performance with respect to the resistance of this sensor is characterized in detail. Meanwhile, properties of the sensor under DC and AC drive current are investigated.

\section{Design and Fabrication}

\subsection{Platinum film structure design and fabrication}

The platinum is designed into a serpentine configuration as the sensitive element in the film sensor. Figure 2 shows the diagram of the sensor. The width and gap of the sensing element are both about $50 \mu \mathrm{m}$, and its thickness is $200 \mathrm{~nm}$. The resistance $R$ of the sensor is calculated using 


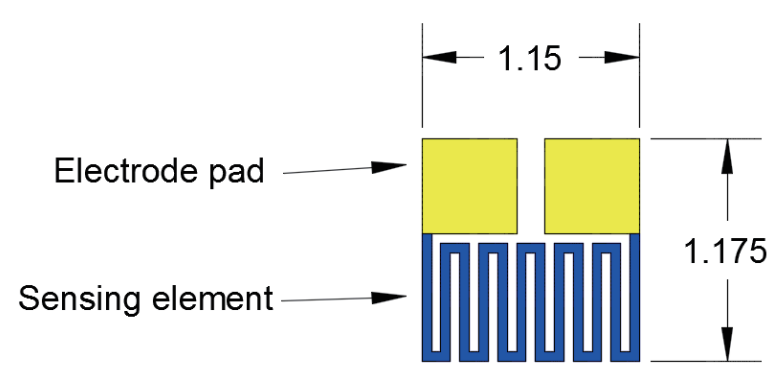

Fig. 2. (Color online) Structure of the platinum sensor.

$$
R=\frac{\rho l}{w t}
$$

where $l$ is the length, $w$ is the width, and $t$ is the thickness of the sensing element. At room temperature, the resistivity $\rho$ is measured as $2.36 \times 10^{-3} \Omega \cdot \mathrm{cm}$ by the four-probe measurement method. Thus, a sensor with a resistance of $182 \Omega$ at room temperature is designed.

Fabrication starts with a standard 3 in silicon wafer as the mechanical support. The fabrication process of the platinum flexible microsensor is presented in Fig. 3. In step (1), polyimide about 50 $\mu \mathrm{m}$ thick was spun and cured. In step (2), the photoresist was coated and patterned. In step (3), a Cr/platinum $(10 \mathrm{~nm} / 200 \mathrm{~nm})$ bilayer was sputtered by DC magnetron sputtering. The Cr film acts as an adhesion layer between the polyimide and the platinum film. The lift-off process was completed to structure the temperature-sensitive element on the polyimide flexible substrate. In step (4), the photoresist was coated and patterned. In step (5), for the realization of bonding pads, a metal bilayer of $\mathrm{Cr} / \mathrm{Au}(10 \mathrm{~nm} / 250 \mathrm{~nm})$ is fabricated by DC magnetron sputtering and a lift-off process. In step (6), the flexible sensors were separated from the silicon substrate. Figure 4(a) shows the image of the flexible microsensor, and Fig. 4(b) shows an optical microscopic image of the micro-temperature sensor.

\subsection{Temperature simulation of polyimide substrate}

The low thermal conductivity of polyimide $[0.15 \mathrm{~W} /(\mathrm{m} \cdot \mathrm{K})]$ will lead to a lower temperature on platinum film and the response time will be long. The finite element analysis software Comsol is used to simulate the temperature distribution and temperature response of the $50-\mu \mathrm{m}$-thick polyimide substrate for a flexible temperature sensor. In the simulation, the temperature of the bottom side of the polymide substrate attached to skin is set as the human-body temperature of $309 \mathrm{~K}$. As the substrate is in contact with air, all the surfaces exposed to ambient are set to have heat flux with the heat transfer coefficient of $5 \mathrm{~W} /\left(\mathrm{m}^{2} \cdot \mathrm{K}\right)$; external temperature is set as 293.15 K. The simulation result are shown in Fig. 5, the temperature difference between the surface attached to skin and the deposited surface platinum film is $0.057 \mathrm{~K}$. To improve the measurement accuracy, the temperature difference is added to the temperature measuerd by the platinum film to compensate the temperature drop caused by the polyimide substrate.

The low thermal conductivity of polyimide also affects the response time of the temperature sensor. Comsol software was used to calculate the response times of temperature sensors on 


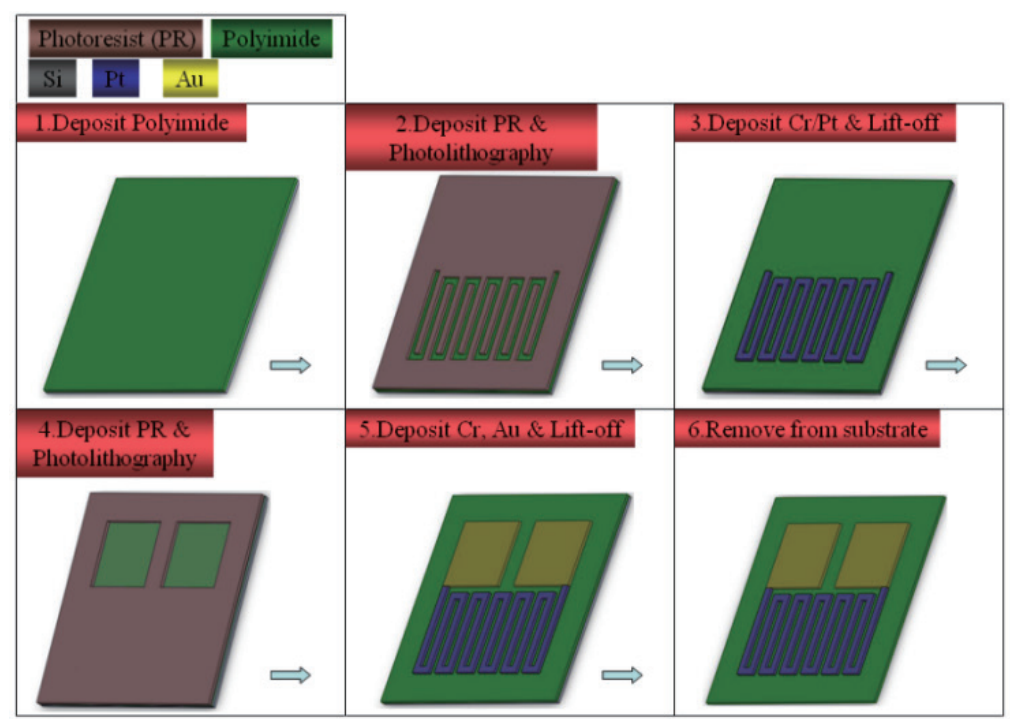

Fig. 3. (Color online) Fabrication process of flexible microsensor.

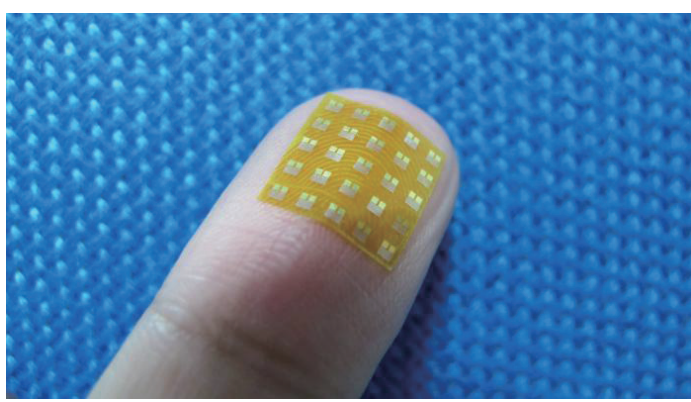

(a)

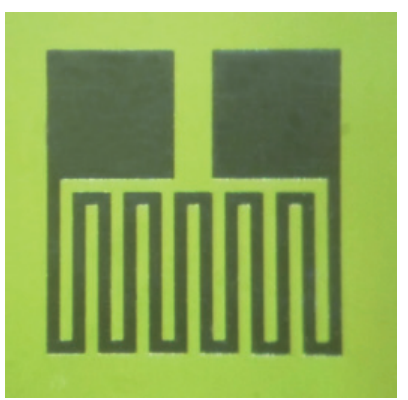

(b)

Fig. 4. (Color online) Fabricated sensor: (a) flexible microsensors and (b) optical microscopic photograph of micro-temperature sensor.

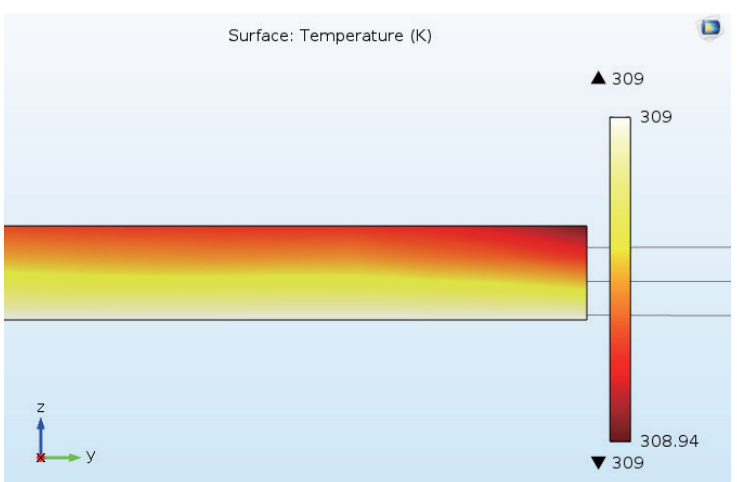

(a)

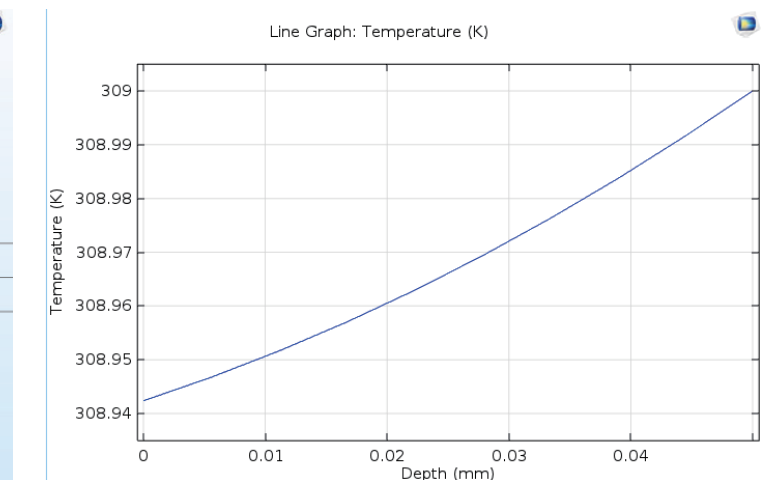

(b)

Fig. 5. (Color online) Temperature simulation of polyimide substrate: (a) temperature distribution along cross section of polyimide substrate and (b) temperature at different depths. 
polyimide, PDMS, and parylene. As can be seen from the simulation results in Fig. 6, the response times of temperature sensors on polyimide, PDMS, and parylene are about 80, 75, and $100 \mathrm{~ms}$, respectively. Temperature sensors on polyimide and PDMS show approximate response times, whereas that on parylene shows a longer response time because of its lower thermal conductivity $[0.1256 \mathrm{~W} /(\mathrm{m} \cdot \mathrm{K})]$.

\section{Characterization of the Sensors}

\subsection{Temperature dependence of resistance}

The testing temperature range to investigate the thermal characterization of the fabricated flexible temperature sensor is from 25 to $100{ }^{\circ} \mathrm{C}$. The resistance under different operating temperatures was recorded using the physical property measurement system (PPMS, Quantum Design Corporation) shown in Fig. 7(a). The fabricated sensors were attached on the sample stage of PPMS during measurement. The temperature fluctuation of the sample stage is controlled to $\pm 0.02 \%$. The accuracy of resistance measurement is more than $0.01 \%$. The testing results are shown in Fig. 7(b).

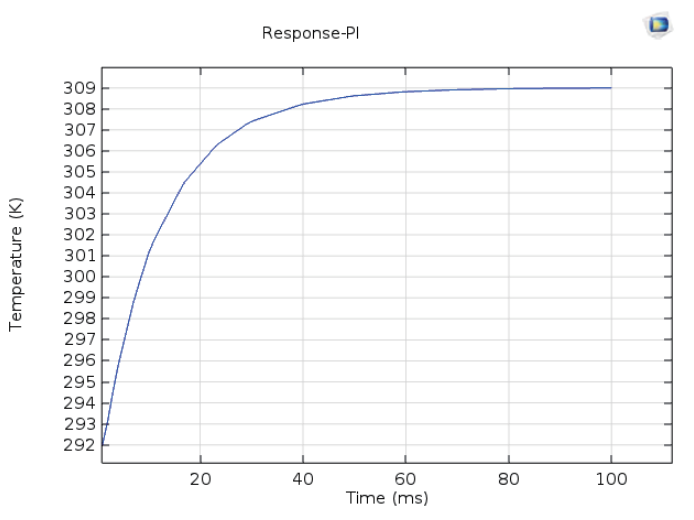

(a)

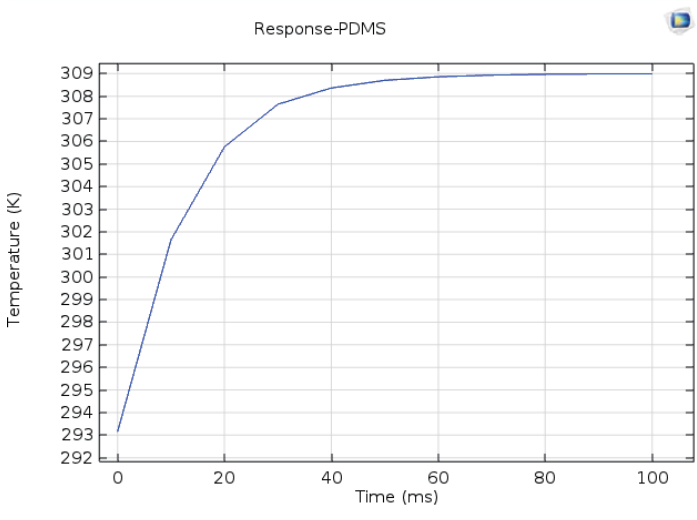

(b)

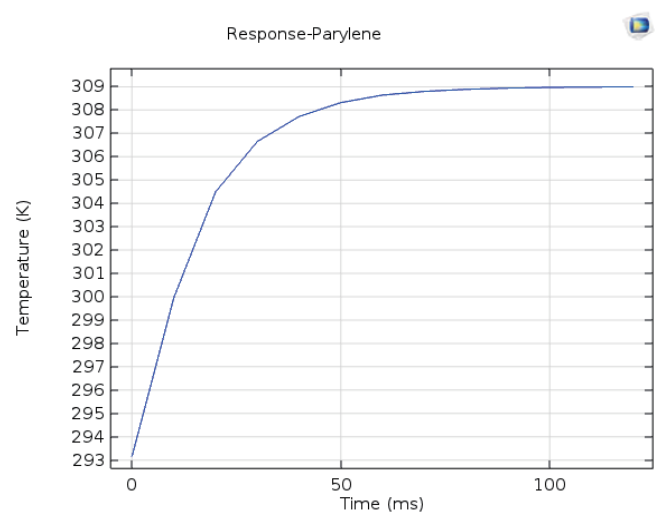

(c)

Fig. 6. (Color online) Temperature transient response: (a) polyimide substratem, (b) PDMS substrate, and (c) parylene substrate. 


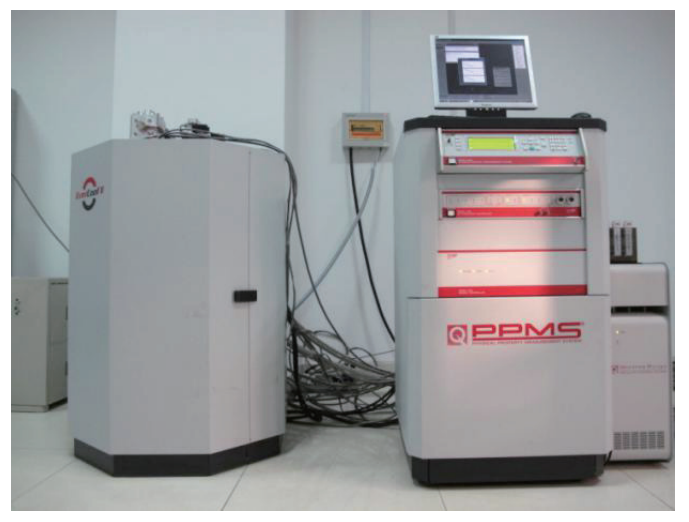

(a)

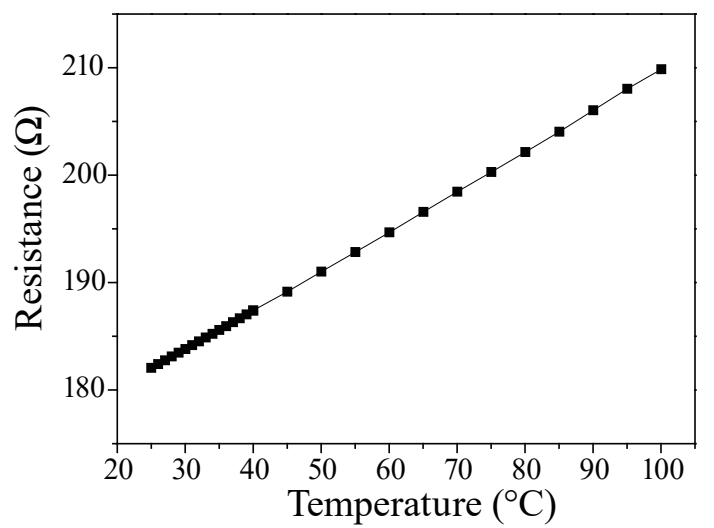

(b)

Fig. 7. (Color online) Resistance variation between 25 and $100{ }^{\circ} \mathrm{C}$ for the flexible sensor: (a) physical property measurement system and (b) test result.

The test data are fitted polynomially, and the result is expressed as

$$
R(T)=-3.57677 \times 10^{-7} T^{3}+3.2739 \times 10^{-4} T^{2}+0.33486 T+173.47695,
$$

where $R(T)$ and $T$ represent the current resistance and temperature of the sensor, respectively. The polynomial is very close to the test data, and the maximum deviation between the polynomial and test data is less than 0.07. It can be seen from the polynomial that the first-degree polynomial plays a dominant role. Thus, there is good linearity between the resistance and temperature. The temperature coefficient of resistance (TCR) between 25 and $100{ }^{\circ} \mathrm{C}$ is expressed as

$$
T C R=\frac{R_{100^{\circ} \mathrm{C}}-R_{25^{\circ} \mathrm{C}}}{R_{25^{\circ} \mathrm{C}} \times\left(100^{\circ} \mathrm{C}-25^{\circ} \mathrm{C}\right)},
$$

where $R_{100}{ }^{\circ} \mathrm{C}$ and $R_{25^{\circ} \mathrm{C}}$ represent the resistances at temperatures of 100 and $25^{\circ} \mathrm{C}$, respectively. On the basis of Eq. (3), TCR of the platinum resistor is $2035 \mathrm{ppm} /{ }^{\circ} \mathrm{C}$, which is less than the value found for bulk platinum resistors $\left(T C R=3850 \mathrm{ppm} /{ }^{\circ} \mathrm{C}\right)$. In the theory of metal electrons, resistance caused by electron scattering at grain boundaries has no relationship with temperature. The crystallinity of platinum film sputtered on polyimide is much lower than that of bulk platinum resistors, and there are more grain boundaries in platinum film. Therefore, the proportion of the resistance of the platinum film attributed to electron scattering at grain boundaries is larger than that of bulk platinum resistors. In other words, the proportion of platinum film resistance independent of temperature is larger than that of bulk platinum resistors. According to Eq. (3), $T C R$ of platinum film is lower than that of bulk platinum resistors.

\subsection{Current-voltage $(I-V)$ curve of flexible sensor}

$I-V$ curve is also used to characterize the thermal properties of the temperature sensor. Power and resistance can be derived from this curve. The output voltage of the sensor is measured under different currents ranging from 0.2 to $40 \mathrm{~mA}$ at $100{ }^{\circ} \mathrm{C}$. As shown in Fig. 8, when the drive current 
is lower than $20 \mathrm{~mA}$, there is a linear relationship between $I$ and $V$. However, the curve deviates from linearity when the drive current is higher than $20 \mathrm{~mA}$. This is because the thermal power has a square relationship with drive current. When the current is high, the self-heating phenomenon is obvious. The temperature of the sensor is changed significantly with increasing thermal power. The platinum film has a positive $T C R$, which causes the resistance of the sensor to increase with increasing drive current. Thus, the upward curve deviates from the straight line. From the measured $T C R$, the increased resistance causes an error in the current temperature. Hence, the drive current for the sensor must be less than $20 \mathrm{~mA}$.

\subsection{Stability of flexible sensor}

The stability of the sensor was evaluated using the resistance variation of the sensor with time when the drive current for the sensor was $20 \mathrm{~mA}$. Figure 9 shows that the resistance of the sensor under a $20 \mathrm{~mA}$ drive current is stable for $120 \mathrm{~min}$ at $100{ }^{\circ} \mathrm{C}$. A slight fluctuation in the curve may be induced by the variation in ambient temperature owing to self-heating. It can be concluded that the sensor is also stable when the drive current is lower than $20 \mathrm{~mA}$ and the ambient temperature is below $100{ }^{\circ} \mathrm{C}$, because self-heating and ambient temperature have minor effects on the sensor.

\subsection{Response time of flexible sensor}

To demonstrate the response time of the sensor, the measurement setup shown in Fig. 10(a) is adopted, the temperature of the heating platform is set at $100{ }^{\circ} \mathrm{C}$, and the flexible sensor is adhered to a glass plate to bring the flexible sensor in contact with the heating platform for convenience. Because the ambient temperature is below $100{ }^{\circ} \mathrm{C}$, the temperature of the sensor quickly reaches $100{ }^{\circ} \mathrm{C}$ when it contacts the heated platform. The variation in the resistance of the sensor is measured using a temperature controller (Model 350 Cryogenic Temperature Controller, LakeShore Cryotronics, electronic accuracy is $\pm 0.004 \Omega$ ). The relationship between time and temperature is derived from the variation in resistance and calculated $T C R$ as

$$
T=\frac{R(T)-R_{25^{\circ} \mathrm{C}}}{\alpha \cdot R\left(T_{25^{\circ} \mathrm{C}}\right)}+25^{\circ} \mathrm{C},
$$

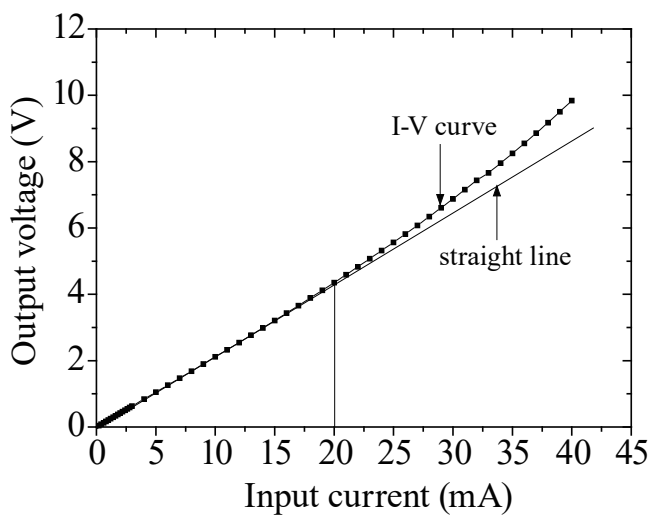

Fig. 8. $I-V$ relationship of the flexible sensor.

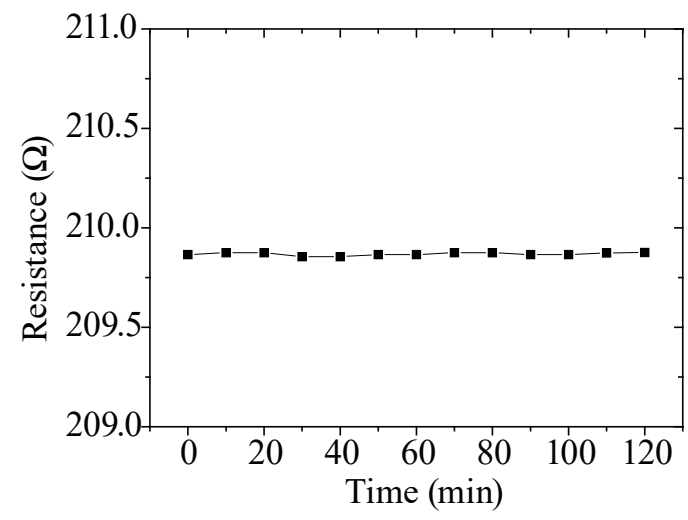

Fig. 9. Evolution of resistance with time at $100^{\circ} \mathrm{C}$. 


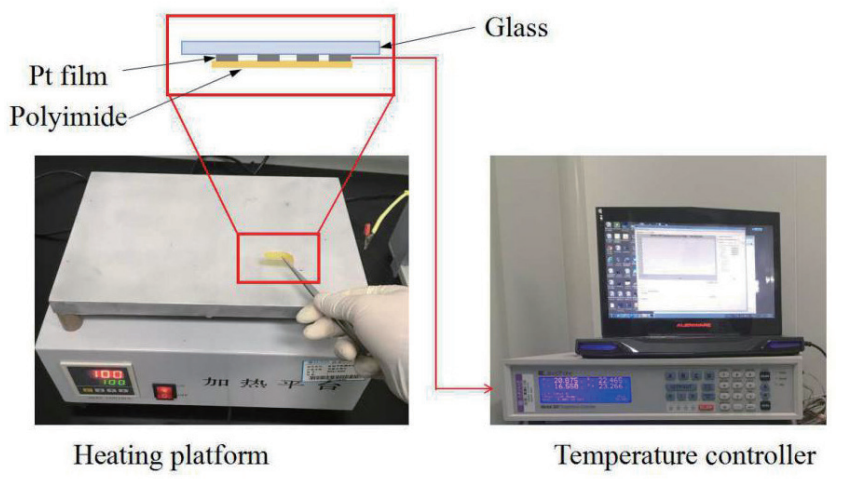

(a)

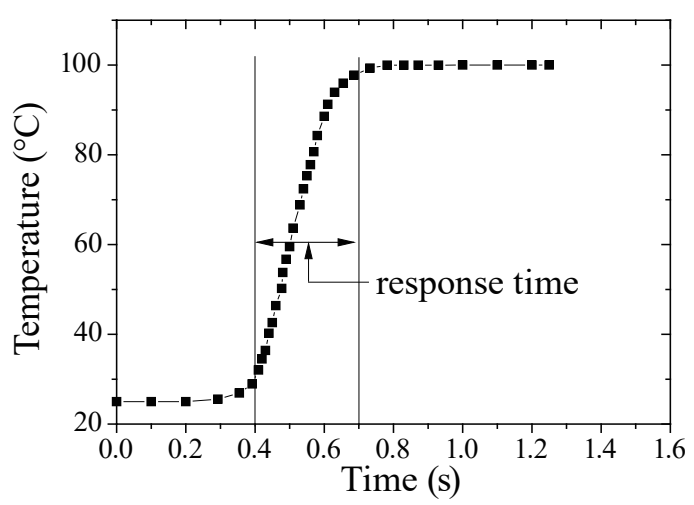

(b)

Fig. 10. (Color online) Temperature response time of the flexible sensor: (a) measurement setup of response time and (b) response of flexible sensor.

where $T, R(T)$, and $\alpha$ are the current temperature, current resistance, and the calculated TCR of the sensor, respectively. The time response of the sensor is shown in Fig. 10(b); it results in a response time of $300 \mathrm{~ms}$. Because the sensor is placed on the heating platform manually, the actual response time of the sensor is far less than $300 \mathrm{~ms}$. The response time is shorter when the platform temperature is lower than $100{ }^{\circ} \mathrm{C}$.

\subsection{Impedance measurement of sensor}

The TCR, $I-V$ curve, and stability of the sensor were measured under DC conditions. The resistances of the sensor under DC at $25,45,65,85$, and $100{ }^{\circ} \mathrm{C}$ are $182.06,189.15,196.57,204.05$, and $209.87 \Omega$, respectively. Because of the compact serpentine structure of the sensor, inductance and capacitance may exist when AC is applied. Thus, it is necessary to study the AC impedance of the sensor.

The AC impedance measurement was performed using an inductance capacitance resistance meter (LCR) analytical instrument in the frequency range from $20 \mathrm{~Hz}$ to $1 \mathrm{MHz}$. The test results show that the impedance increased slightly with increasing AC frequency, as shown in Fig. 11.

When the AC frequency is within $100 \mathrm{kHz}$, the maximum absolute deviation between impedance and resistance is less than $0.4 \Omega$. The test of the dependence of resistance on temperature shows that when the temperature is increased $1{ }^{\circ} \mathrm{C}$, the resistance increases $0.4 \Omega$. Thus, the corresponding temperature error is controlled within $1^{\circ} \mathrm{C}$. However, when the frequency is as high as $1 \mathrm{MHz}$, the deviation between impedance and resistance is larger than $0.4 \Omega$ for the given temperatures. It can be concluded that the $\mathrm{AC}$ frequency has little effect on the impedance of the sensor when the AC frequency is less than $100 \mathrm{kHz}$. In other words, the sensor under $\mathrm{AC}$ drive current within an $\mathrm{AC}$ frequency of $100 \mathrm{kHz}$ has the same electrical properties as that under DC drive current. 


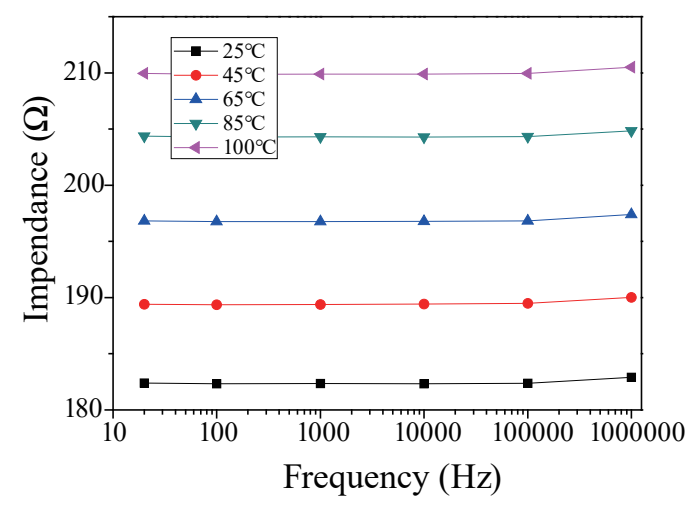

Fig. 11. (Color online) Impedance versus frequency as a function of temperature.

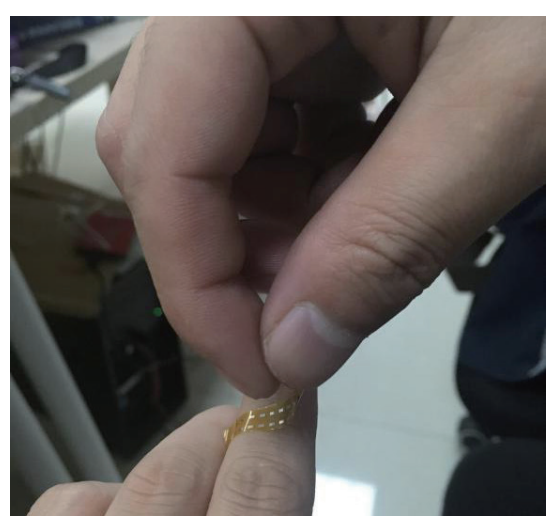

(a)

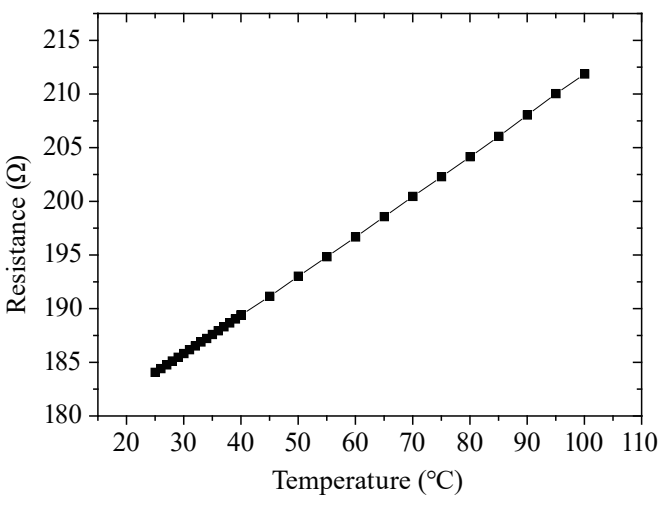

(b)

Fig. 12. (Color online) Bending experiment: (a) flexible temperature sensor attached to finger and (b) results for flexible temperature sensor stripped off finger.

\subsection{Adhesion stability test}

To test the adhesion stability, a fabricated flexible sensor array is attached to and stripped off the skin of a human finger 100 times, as shown in Fig. 12. The metal film on polyimide showed good adhesion to the substrate during the bending experiment. The resistance of the flexible sensor under different operating temperatures was recorded using the PPMS after the bending test. On the basis of Eq. (3), TCR of the platinum resistor after the bending test is $0.002014{ }^{\circ} \mathrm{C}^{-1}$, which is almost the same as the $T C R$ of the flexible sensor before bending.

\section{Conclusions}

The feasibility of a microfabrication process for realizing a platinum flexible microsensor for the measurement of temperature distribution has been demonstrated. Polyimide is used as the substrate of the sensor, because it has a high ultrasonic propagation rate and is resistant to high 
temperature. The dependence of resistance on temperature of the sensor was measured over a temperature range from 25 to $100{ }^{\circ} \mathrm{C}$, which covers the range of HIFU applications. The sensor is highly sensitive to temperature and has a high $T C R$ of $2035 \mathrm{ppm} /{ }^{\circ} \mathrm{C}$. When the drive current is lower than $20 \mathrm{~mA}$, the sensor is not affected by self-heating, and it will respond sensitively to the current temperature. The sensor has high stability and a response time of $0.3 \mathrm{~s}$. The impedance results show there is little difference in the properties between $\mathrm{AC}$ and $\mathrm{DC}$ drive current when the $\mathrm{AC}$ frequency is below $100 \mathrm{kHz}$. This performance shows the flexible sensor is perfectly suited to HIFU applications.

\section{Acknowledgments}

This work was supported in part by the National Natural Science Foundation of China (51565038), the Science and Technology Project of Jiangxi Provincial Department of Science and Technology (20122BBE500033, 20151BBE50044, and 2016BAB216106) and project of Jiangxi Provincial Department of Education (GJJ15112).

\section{References}

1 J. G. Lynn and T. J. Putnam: Am. J. Path. 20 (1994) 3.

2 G. R. Ter Haar: Echocardiography 18 (2001) 4.

3 S. Dasguplatinuma, R. K. Banerjee, P. Hariharan, and M. R. Myers: Ultrasonics 51 (2011) 2.

4 C. Y. Lee, G. W. Wu, and W. J. Hsieh: Sens. Actuators, A 147 (2008) 1.

5 T. Q. Trung, S. Ramasundaram, B. U. Hwang, and N. E. Lee: Adv. Mater. 28 (2016) 3.

6 S. Ali, A. Hassan, J. Bae, C. H. Lee, and J. Kim: Langmuir 32 (2016) 44.

7 Z. Z. Wu, C. Y. Li, J. Hartings, S. Ghosh, R. Narayan, and C. Ahn: J. Micromech. Microeng. 27 (2016) 0025001

8 J. S. Park, D. S. Lee, H. W. Nho, D. S. Kim, T. H. Hwang, and N. K. Lee: Sens. Mater. 29 (2017) 1275.

9 R. R. Richardson, J. A. Miller, and W. M. Reichert: Biomaterials 14 (1993) 8.

10 J. M. Seo, S. J. Kim, H. Chung, E. T. Kim, H. G. Yu, and Y. S. Yu: Mater. Sci. Eng. 24 (2004) 1.

11 Y. Moser and A. M. Gijs: J. Microelectromech. Syst. 16 (2007) 6.

12 B. Hadimioglu and B. T. Khuri-Yakub: IEEE Ultrasonics Symp. 3 (1990) 3.

13 D. Resnik, D. Vrtačnik, U. Aljančič, M. Možek, B. Pečar, S. Penič, and S. Amon: Industrial Electronics, IECON '09. 35th Ann. Conf. IEEE (Porto, Portugal, 2009) p. 4014.

14 S. Sedky, A. Witvrouw, H. Bender, and K. Baert: IEEE Trans. Electron Devices 48 (2001) 2.

15 A. Rajagopala, S. Walavalkar, S. Chen, L. Guo, and T. Gwinn: Appl. Phys. Lett. 97 (2010) 133109.

16 S. Metz, A. Bertsch, D. Bertrand, and P. Renaud: Biosens. Bioelectron. 19 (2004) 10.

17 T. Stieglitz, M. Schuettler, and K. P. Koch: Eng. Med. Biol. Mag. 24 (2005) 5.

18 D. Briand, S. Colin, A. Gangadharaiah, E. Vela, P. Dubois, L. Thiery, and N. F. de Rooij: Sens. Actuators, A 132 (2006) 1.

19 J. Han, Z. Tan, K. Sato, and M. Shikida: J. Micromech. Microeng. 15 (2005) 2.

20 S. A. Dayeh, D. P. Butler, and Z. Celik-Butler: Sens. Actuators, A 118 (2005) 1. 